> La survie d'une espèce dépend de deux processus intimement liés : la reproduction, d'une part, et l'équilibre entre les besoins énergétiques et l'approvisionnement en sources d'énergie par l'alimentation, d'autre part. Ces deux processus sont contrôlés dans le cerveau par l'hypothalamus, qui produit des neurohormones agissant sur l'hypophyse pour piloter diverses fonctions physiologiques. L'une de ces neurohormones, la $\mathrm{GnRH}$, contrôle non seulement la maturation et le fonctionnement des organes reproducteurs, incluant les ovaires et les testicules, lors de la puberté et à l'âge adulte, mais aussi l'attirance sexuelle. De récentes découvertes suggèrent que la signalisation impliquant la neuropiline- 1 dans les neurones sécrétant la GnRH jouerait un rôle charnière dans la coordination du neurodéveloppement et des adaptations physiologiques et comportementales nécessaires au déclenchement de la puberté et à l'acquisition de la fonction de reproduction. Dans cet article de synthèse, nous replaçons ces découvertes dans le contexte de récents travaux montrant que les voies de signalisation des sémaphorines de classe 3 sont impliquées dans la physiopathologie non seulement de l'infertilité, mais aussi de l'obésité. Nous discutons également l'implication potentielle des neurones produisant la GnRH dans la perception des odeurs sociales et dans la précocité de la maturation sexuelle. L'hypothèse selon laquelle l'activité de ces neurones au cours du développement postnatal constituerait le chaînon manquant entre la prise de poids, le déclenchement de la puberté et le comportement sexuel, ouvre la voie à une meilleure compréhension de l'implication de l'homéostasie énergétique dans la maturation sexuelle, et pourrait aussi avoir des implications thérapeutiques pour la puberté précoce. <

\section{Signalisation impliquant la neuropiline dans les neurones sécrétant la GnRH}

Son rôle dans

le déclenchement

de la puberté

Charlotte Vanacker, Sébastien G. Bouret, Paolo Giacobini, Vincent Prévot

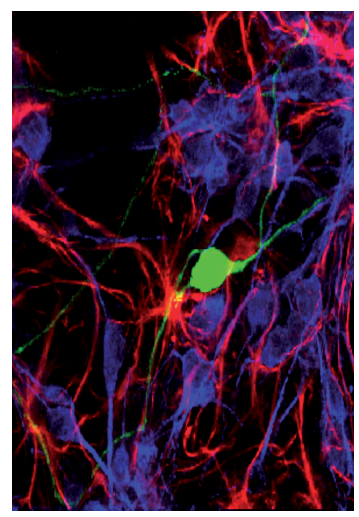

Univ. Lille, Inserm, CHU Lille, Équipe développement et plasticité du cerveau neuroendocrine, FHU 1000 jours pour la Santé, Lille Neuroscience et Cognition, UMR-S1172, 1 place de Verdun, 59045 Lille Cedex, France. vincent.prevot@inserm.fr

La survie d'une espèce dépend, pour ses individus, de deux impératifs : le maintien de l'homéostasie énergétique et la capacité de transmettre du matériel génétique à des descendants. Chez les mammifères, animaux à reproduction sexuée, ces processus sont contrôlés dans le cerveau par deux circuits hypothalamiques différents. La puberté, puis, à l'âge adulte, la fertilité, sont sous le contrôle de l'axe hormonal hypothalamo-hypophyso-gonadique. Elles sont orchestrées par une petite population de cellules neuroendocrines de l'hypothalamus, qui communiquent avec l'hypophyse en sécrétant la gonadolibérine (gonadotropin-releasing hormone, GnRH) dans la circulation porte hypothalamo-hypophysaire ${ }^{1}$. La GnRH stimule la sécrétion de l'hormone lutéinisante (LH) et de l'hormone folliculo-stimulante (FSH) par les cellules gonadotropes de l'hypophyse. Ces hormones gonadotropes contrôlent à leur tour, dans les deux sexes, la synthèse des stéroïdes sexuels et la gamétogenèse dans les gonades : testicules chez le mâle, ovaires chez la femelle [1, 2] (Figure 1).

La puberté, marquée par le développement des caractères sexuels corporels et certains changements comportementaux, s'achève avec l'acquisition de la fertilité, c'est-à-dire la capacité de l'individu à avoir une reproduction sexuée. C'est un processus contrôlé par un

Vignette (Photo @ Inserm-Prévot, Vincent)

${ }^{1}$ Système vasculaire reliant l'hypothalamus et l'antéhypophyse. 


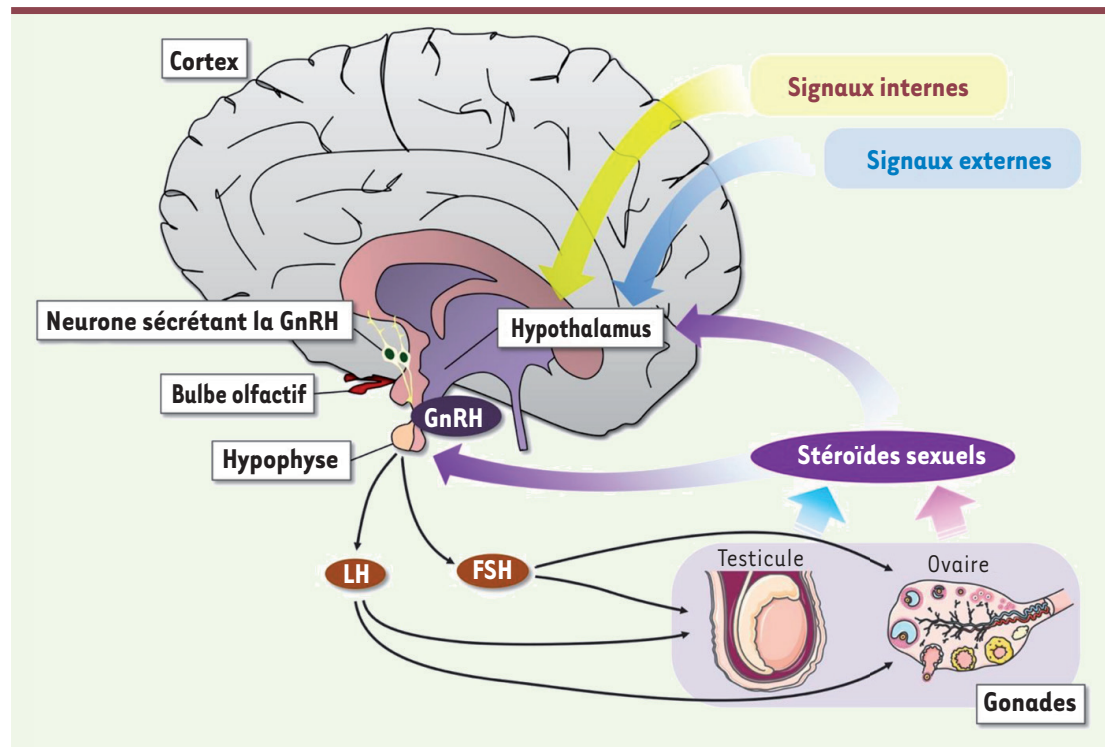

Figure 1. L'axe hormonal hypothalamo-hypophyso-gonadique.

ensemble complexe de déterminants génétiques et environnementaux. L'accélération de la croissance dans la période pré-pubertaire est considérée comme un facteur permissif important du déclenchement de la puberté dans l'espèce humaine [2, 3]. Chez les patients atteints d'hypogonadisme hypogonadotrope (une cause d'infertilité d'origine hypothalamique) à cause d'un arrêt de la migration des neurones produisant la GnRH entre le nez et le cerveau au cours de l'embryogenèse, ou à cause d'un dysfonctionnement de ces neurones dans l'hypothalamus, on observe une absence de puberté spontanée [1]. D'autres individus souffrent au contraire d'une puberté précoce, dont les causes restent très mal connues.

\section{Facteurs chimiotropes dans le développement des neurones produisant la $\mathrm{GnRH}$}

Au cours du développement embryonnaire, les neurones sécréteurs de GnRH migrent depuis la cavité nasale vers le cerveau, le long des fibres des nerfs olfactifs et des nerfs terminaux $[4,5]$. Leur migration est contrôlée par des signaux chimiques diffusibles [6]. La famille des sémaphorines est l'une des plus importantes familles de facteurs chimiotropes. Très conservée au cours de l'évolution, elle joue notamment un rôle essentiel dans la formation des réseaux neuronaux. Les neurones sensoriels olfactifs expriment la sémaphorine- $3 \mathrm{~A}$, qui se lie, sur leurs axones, à un récepteur membranaire de type plexine en présence du corécepteur neuropiline-1 [5-7] (Figure 2). La sémaphorine-3A et la neuropiline- 1 sont impliquées dans le codage spatial des informations sensorielles dans les bulbes olfactifs [7]. Durant l'embryogenèse, les axones des neurones sensoriels olfactifs, qui expriment la neuropiline-1, forment également l'échafaudage le long duquel les neurones produisant la GnRH migrent vers le cerveau [8] (Figure 3). $\varepsilon n$ étudiant, chez la souris, le rôle de la neuropiline-1 produite par les neurones synthétisant la GnRH, nous avons montré que leur migration en nombre excessif vers l'hypothalamus et l'accélération de leur maturation dans le cerveau pouvait entraîner une puberté précoce, mais aussi une attirance prématurée pour les odeurs de l'autre sexe, et, étonnamment, un surpoids. Ces observations suggéraient l'implication de ces neurones dans le contrôle du métabolisme énergétique et de l'accélération pré-pubertaire de la croissance [9].

\section{Rôle de la neuropiline-1 dans la migration des neurones produisant la GnRH}

Des mutations hétérozygotes touchant des gènes de la voie de signalisation des sémaphorines de classe 3 ont été identifiées chez des patients présentant un déficit congénital en GnRH (hypogonadisme hypogonadotrope congénital) [10-12] et chez certains patients souffrant d'une obésité précoce sévère [13]. Cependant, contrairement aux patients atteints d'hypogonadisme hypogonadotrope et porteurs de mutations du gène codant la neuropiline-l, ou aux souris mutantes chez lesquelles toutes les cellules qui expriment normalement ce gène ne l'expriment plus $[10,11]$ (Figure 3), les animaux rendus génétiquement déficients en neuropiline- 1 uniquement dans les neurones produisant la GnRH n'ont pas de déficit en GnRH, et présentent au contraire une maturation prématurée de ces neurones et une puberté précoce [9] (Figure 4). Cette apparente contradiction pourrait s'expliquer par le fait qu'un déficit ubiquitaire de ta neuropiline-l, tant chez l'homme que chez la souris, modifie les projections des fibres des nerfs olfactifs et terminaux pendant la période embryonnaire de migration des neurones produisant la GnRH, ce qui perturbe cette migration [10, 11] (Figure 3), tandis que l'invalidation sélective du gène de la neuropiline-l dans les neurones produisant la GnRH augmente leur survie et donc leur nombre, accélère leur migration pendant la période embryonnaire, et altère leur intégration dans les réseaux hypothalamiques contrôlant leur activité après la naissance [9] (Figure 4). En effet, la signalisation impliquant la neuropiline-1, non seulement joue un rôle de guidage axonal lors du développement [7], mais aussi intervient dans la mort cellulaire programmée (apoptose) [14]. L'effet anti-apoptotique de la suppression ciblée de cette signalisation dans les neurones synthétisant la GnRH chez les souris mutantes, qui peut être mimé par l'injection, dans le nez des embryons, d'anticorps neu- 
tralisant la neuropiline-1 ou d'un composé anti-apoptotique in utero, entraîne la présence d'un plus grand nombre de ces neurones dans la région préoptique de l'hypothalamus, où ils résident habituellement, mais aussi dans les bulbes olfactifs accessoires, où ils sont habituellement très peu nombreux [9].

\section{Rôle de la signalisation impliquant la neuropiline-1 dans les neurones sécrétant la GnRH sur le déclenchement de la puberté}

Chez la souris, le déficit en neuropiline-l dans les neurones produisant la $\mathrm{GnRH}$ accélère la maturation de leur activité électrique au cours du développement postnatal dans les deux sexes [9]. Cet effet est vraisemblablement lié à un défaut de l'intégration de ces neurones dans les réseaux de neurones afférents, peut-être par un déficit de stabilisation des contacts synaptiques qui nécessitent la voie de signalisation des sémaphorines [15]. Ce phénomène, associé à une présence surnuméraire de ces neurones dans l'hypothalamus, pourrait expliquer la précocité pubertaire dans ce modèle animal. Les résultats d'une étude de génétique humaine suggèrent en effet que la cinétique de la puberté dépend du nombre de neurones produisant la GnRH qui ont atteint l'hypothalamus au cours de la vie embryonnaire [16].

\section{Facteurs génétiques contrôlant l'âge de la puberté et la prise de poids}

Dans l'espèce humaine, même si l'on considère actuellement que l'âge de la puberté est principalement contrôlé par des facteurs génétiques $[2,3]$, les facteurs déterminants de son déclenchement sont pour la plupart inconnus. À ce jour, le gène le plus fréquemment muté chez les individus présentant une puberté précoce à caractère héréditaire est MKRN3 (makorin RING finger protein 3) [17], dont l'expression hypothalamique décroît physiologiquement au cours du développement postnatal, à la fois chez les primates et chez les rongeurs [18]. Chez le rat femelle, l'altération de l'inhibition de l'expression de Mkrn3 dans l'hypothalamus au cours du développement post-natal provoque d'ailleurs une puberté retardée [19]. D'autres gènes dont l'expression est modulée au cours du développement peuvent être mutés chez ces individus: DLKI (delta-like non-canonical Notch ligand 1), muté chez des patients qui présentent également un surpoids ou une obésité [20], et KISSI (codant la kisspeptine) [21] ou GPR54 (codant le récepteur de la kisspeptine) [22], deux gènes dans lesquels on trouve également des mutations chez des patients atteints d'hypogonadisme hypogonadotrope congénital [1]. Le fait que la suppression génétique de la neuropiline-l dans les neurones produisant la GnRH entraîne une puberté précoce chez la souris suggère que certains variants nucléotidiques rares du gène codant ce corécepteur et, plus généralement, de gènes codant des protéines impliquées dans la voie de signalisation de la sémaphorine-3A, pourraient être présents dans l'espèce humaine chez des individus exprimant ce trait phénotypique [9]. Des variants nucléotidiques des gènes SEMA3A (codant la sémaphorine-3A) et NRPI (codant la neuropiline-1), mais aussi des gènes PLEXIN Al-4 (codant

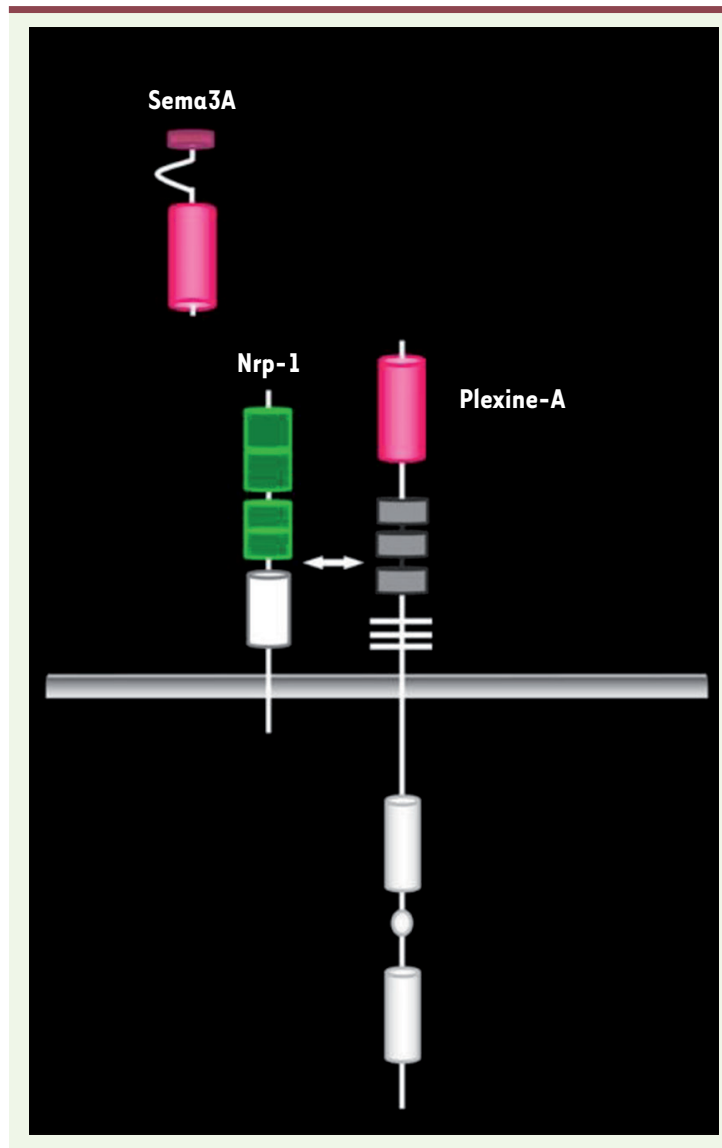

Figure 2. Voie de signalisation sémaphorine 3A/neuropiline 1/ plexine A. La sémaphorine 3A (Sema3A) se lie au récepteur plexineA et au co-récepteur neuropiline-1 (Nrp-1) pour activer des voies de signalisation intracellulaires effectrices (d'après [40-42]).

les plexines 1 à 4), ont récemment été identifiés chez des patients présentant une forme grave d'obésité dès l'enfance [13], un phénotype qui serait associé à un développement défectueux du circuit mélanocortinergique. La perturbation de la signalisation de la sémaphorine-3A dans les neurones anorexigènes exprimant la pro-opiomélanocortine (POMC) diminue la capacité de ces derniers à établir des connexions avec leurs régions cérébrales cibles lors du développement postnatal, et entraîne une prise de poids modérée [13]. Nous avons également montré l'existence d'un surpoids modéré chez les souriceaux femelles dont le gène de la neuropiline-1 a été invalidé spécifiquement dans les neurones produisant la GnRH [9], ce qui suggère que ces neurones jouent un rôle dans le contrôle de l'homéostasie énergétique au cours du développement postnatal. Il serait intéressant de déterminer, de manière rétrospective, si, chez l'homme, les individus atteints d'obésité dans l'enfance et portant une 


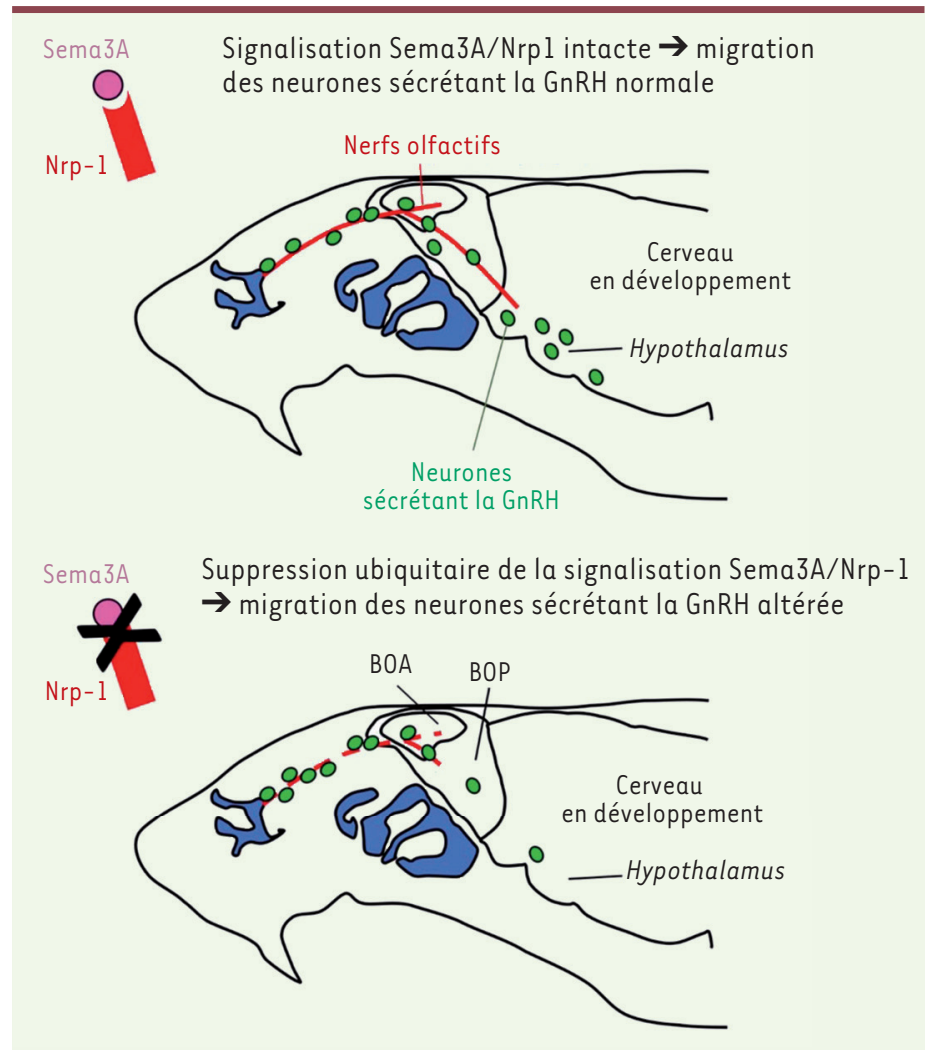

Figure 3. Rôle de la voie de signalisation sémaphorine 3A/neuropiline 1 dans la migration axophilique des neurones sécrétant la GnRH. Pendant la vie embryonnaire, les neurones produisant la GnRH (colorés en vert) migrent du nez vers le cerveau le long des fibres des nerfs olfactifs et olfactifs accessoires (en rouge). Chez les souris mutantes dépourvues de signalisation sémaphorine-3A/plexine-A/ neuropiline-1, le développement de ces nerfs est anormal et les neurones produisant la GnRH n'atteignent pas leur destination normale dans la région hypothalamique du cerveau. BOP : bulbe olfactif principal ; BOA : bulbe olfactif accessoire.

mutation d'un gène codant une protéine impliquée dans la voie de signalisation de la sémaphorine-3A [13] ont eu une puberté précoce d'origine centrale?

\section{Puberté et dimorphisme sexuel}

L'accélération de la croissance pendant la période prépubertaire, qui se traduit par une augmentation de la masse grasse chez les filles et de la masse maigre chez les garçons [23], est considérée comme un facteur permissif de la maturation pubertaire [24, 25]. Cependant, chez les souris femelles dont le gène de la neuropiline-l a été invalidé spécifiquement dans les neurones produisant la GnRH, la prise de poids et l'augmentation de la masse grasse dès le sevrage, qui résulte de la maturation précoce de l'activité de ces neurones [9], semble indi-

\footnotetext{
2 Les pubertés précoces centrales sont dues à la réactivation prématurée de l'axe hormonal hypothalamo-hypophyse-gonades. Elles se distinguent des pubertés précoces d'origine périphérique, qui ont pour cause une atteinte ovarienne ou testiculaire, ou sont secondaires à une tumeur sécrétant l'hormone chorionique gonadotrope.
}

quer que c'est l'activation centrale de l'axe hormonal hypothalamo-hypophyso-gonadique qui déclenche la poussée de croissance en fin de période juvénile, et non l'inverse. Une augmentation prématurée de la masse grasse et la constatation d'un indice de masse corporelle plus élevé chez la fille pourraient donc indiquer l'activation précoce des neurones produisant la GnRH et annoncer la survenue d'une puberté précoce d'origine centrale $[2,26]$.

Dans ce modèle murin de puberté précoce par invalidation ciblée du gène de la neuropiline-l dans les neurones synthétisant la GnRH [9], la chronologie de la puberté n'a pas été examinée chez les mâles mutants en raison du caractère invasif de la procédure expérimentale nécessaire pour détecter la présence de spermatozoïdes dans la jonction entre l'épididyme et le canal déférent [27]. La durée de maturation des spermatozoïdes, après activation de l'axe hypothalamo-hypophyso-gonadique pendant la période infantile, est relativement incompressible chez la souris [28], ce qui réduit considérablement les possibilités d'intervention. $\varepsilon$ n effet, la spermatogenèse est un long processus ( $40-50$ jours chez les rongeurs) et la souris mâle est capable d'engendrer une descendance dès l'âge de 6-7 semaines (âge de la puberté chez le mâle) [24, 29]. Cependant, étant donné l'augmentation du nombre de neurones synthétisant la GnRH dans l'hypothalamus et leur activation précoce chez les animaux mutants des deux sexes, il est probable que les mâles mutants aient, comme les femelles mutantes, une puberté précoce, même si cela reste à démontrer.

\section{Neuropiline-1, GnRH et prématurité sexuelle}

Un autre facteur qui pourrait contribuer à la puberté précoce chez les souris rendues génétiquement déficientes en neuropiline-l dans les neurones produisant la GnRH, est l'accumulation anormale de ces neurones dans les bulbes olfactifs accessoires [9], où leur nombre est habituellement faible chez l'homme et chez les rongeurs [8] (ce qui résulte vraisemblablement de l'effet répulsif de la forte expression de sémaphorine$3 \mathrm{~A}$ dans cette région pendant l'embryogenèse [30]). Chez le campagnol, l'augmentation des taux de GnRH dans les bulbes olfactifs accessoires a été associée à une libération de LH dans la circulation générale lors de stimulations phéromonales (impliquées dans l'attirance sexuelle) [31]. Chez la souris, les signaux déclenchés par les phéromones ${ }^{3}$ dans les neurones sen-

\footnotetext{
3 Une phéromone est une substance chimique volatile produite par une glande exocrine (et non pas endocrine comme pour les hormones), qui agit comme un message olfactif entre les individus d'une même espèce et, dans le cas des phéromones impliquées dans la fonction de reproduction, entre individus de sexes différents.
} 


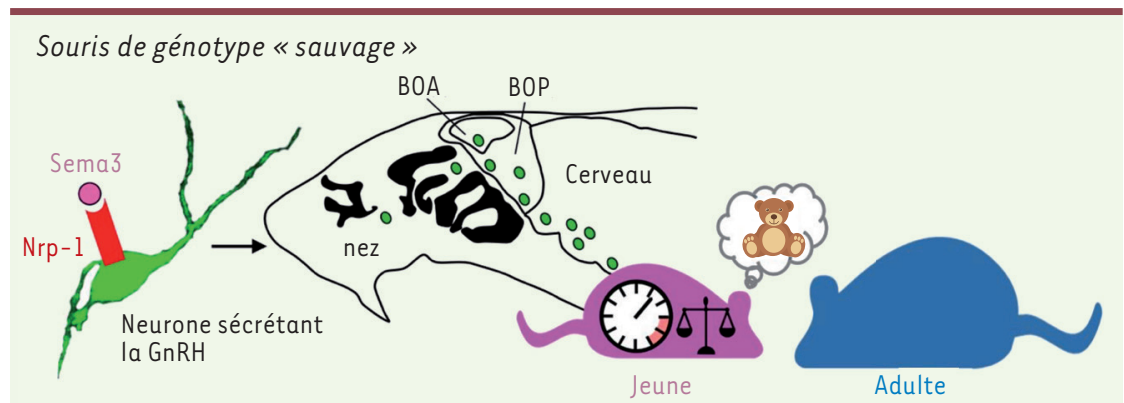

Souris mutante n'exprimant plus le corécepteur Nrp-1 des protéines Sema3 dans les neurones sécrétant la GnRH

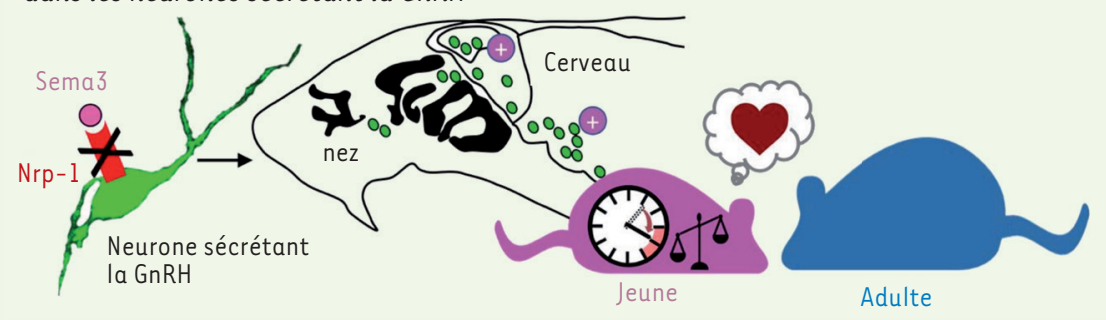

Figure 4. Rôle de l'expression de neuropiline 1 dans les neurones sécrétant la GnRH sur leur migration durant la période embryonnaire et sur la maturation de la fonction de reproduction. L'expression de la neuropiline-1 ( $\mathrm{Nrp}-\mathrm{l})$ dans les neurones produisant la GnRH (colorés en vert) est impliquée dans le contrôle de leur migration, de leur survie, et de leur activité, et joue un rôle dans l'attirance sexuelle (bulle), la prise de poids prépubère (taille de la souris mauve) et le moment de la puberté (horloge). BOP: bulbe olfactif principal; BOA : bulbe olfactif accessoire.

$80 \%$ des cas), sont en effet exposés à un risque plus élevé d'abus sexuel ou de début précoce d'activité sexuelle [36]. Des études d'imagerie réalisées chez l'Homme ont montré que le traitement des individus par la kisspeptine, qui

soriels de l'organe voméro-nasal sont transmis, par voie nerveuse, non seulement aux neurones des bulbes olfactifs accessoires, mais aussi aux neurones sécrétant la GnRH, qui peuvent eux-mêmes influencer le traitement de ces informations olfactives par des boucles de rétroaction [32]. Une étude récente montre que l'expression de la GnRH est nécessaire pour déterminer la préférence sexuelle chez la souris femelle adulte [33]. Afin d'exclure un rôle potentiel des stéroïdes gonadiques (dont la sécrétion, rappelons-le, est dépendante de la $\mathrm{GnRH}$ ) dans ce processus, cette étude, qui montre l'importance des neurones produisant la $\mathrm{GnRH}$ dans le traitement des odeurs sociales, a été conduite chez des souris subissant une perte de l'activité du promoteur du gène codant la GnRH pendant le développement postnatal [34] après gonadectomie [33]. Dans notre modèle murin de puberté précoce, environ la moitié des souriceaux mutants de sexe féminin n'exprimant pas la neuropiline-l dans les neurones sécrétant la GnRH sont attirés précocement, dès le sevrage, par l'urine de mâles sexuellement matures [9], alors que ce type de préférence sexuelle ne s'observe normalement que plus tard, après la puberté, lorsque les paramètres neuroendocriniens et comportementaux de la maturation sexuelle s'accordent pour répondre efficacement aux demandes physiologiques [35]. Une libération anormalement élevée de GnRH dans les bulbes olfactifs accessoires pourrait donc être responsable des modifications de la perception et du traitement des informations olfactives à valeur sexuelle au cours du développement postnatal chez ces souris femelles mutantes [9].

Ces observations renforcent l'hypothèse d'une implication directe des cellules neuroendocrines contrôlant la reproduction dans le comportement sexuel. Elles fournissent également un cadre théorique permettant de mieux comprendre les fondements biologiques des risques psychosociaux ou de comportements inadaptés chez les enfants ayant une puberté précoce par activation prématurée du système neuroendocrinien de la reproduction. Ces enfants, en majorité des filles (60 à active la libération de $\mathrm{GnRH}$, améliore les réponses aux stimulus sexuels et réduit l'aversion sexuelle [37]. Une piste intéressante pour de futures recherches serait de déterminer si, en plus d'entraîner des troubles du comportement, la maturation précoce de l'axe hormonal hypothalamo-hypophyso-gonadique chez les juvéniles de sexe féminin n'altère pas la production de signaux chimiques bloquant, chez la souris, les approches sexuelles des jeunes femelles par les mâles matures [38], et chez l'homme, l'excitation sexuelle [39]. $\diamond$

\section{REMERCIEMENTS}

Ces travaux ont reçu le soutien financier de la métropole européenne de Lille (MEL, No : Convention_2017_ESR_04), de l'agence nationale de la recherche (ANR-14-CE12-0015 et ANR-17-CE16-0015) et de l'EU COST action (BM1105).

\section{LIENS D'INTÉRÊT}

Les auteurs déclarent n'avoir aucun lien d'intérêt concernant les données publiées dans cet article.

\section{SUMMARY}

Precocious puberty and neuropilin- 1 signaling in GnRH neurons

The survival of the species depends on two closely interlinked processes: the correct functioning of the reproductive system, and the balance between the energy needs of an individual and the supply of energy sources through feeding. These two processes are regulated in the hypothalamus, which produces neurohormones that control various physiological functions. Among these neurohormones, GnRH controls not only the maturation and function of the reproductive organs, including 
the ovaries and the testes, during puberty and in adulthood, but also sexual attraction. Recent evidence suggest that neuropilin-1-mediated signaling in $\mathrm{GnRH}$-synthesizing neurons could be a linchpin that holds together various neuroanatomical, physiological and behavioral adaptations involved in triggering puberty and achieving reproductive function. $\diamond$

\section{RéFÉRENCES}

1. Boehm U, Bouloux PM, Dattani MT, et al. Expert consensus document: European consensus statement on congenital hypogonadotropic hypogonadism--pathogenesis, diagnosis and treatment. Nat Rev Endocrinol 2015 ; 11 : 547-64.

2. Howard SR, Dunkel L. Delayed puberty-phenotypic diversity, molecular genetic mechanisms, and recent discoveries. Endocr Rev $2019 ; 40: 1285-317$.

3. Parent AS, Teilmann G, Juul A, et al. The timing of normal puberty and the age limits of sexual precocity: variations around the world, secular trends, and changes after migration. Endocr Rev $2003 ; 24: 668-93$.

4. Schwanzel-Fukuda M, Pfaff DW. Origin of luteinizing hormone-releasing hormone neurons. Nature $1989 ; 338: 161-4$

5. Wray $\mathrm{S}$, Grant $\mathrm{P}$, Gainer $\mathrm{H}$. Evidence that cells expressing luteinizing hormone-releasing hormone mRNA in the mouse are derived from progenitor cells in the olfactory placode. Proc Natl Acad Sci USA $1989 ; 86: 8132-6$.

6. Giacobini P. Shaping the reproductive system: role of semaphorins in gonadotropin-releasing hormone development and function. Neuroendocrinology $2015 ; 102: 200-15$.

7. Imai T, Yamazaki T, Kobayakawa R, et al. Pre-target axon sorting establishes the neural map topography. Science $2009 ; 325: 585-90$.

8. Casoni F, Malone SA, Belle M, et al. Development of the neurons controlling fertility in humans: new insights from 3D imaging and transparent fetal brains. Development 2016; 143 : 3969-81.

9. Vanacker C, Trova S, Shruti S, et al. Neuropilin-1 expression in GnRH neurons regulates prepubertal weight gain and sexual attraction. EMBOJ 2020 ; 39 : e104633.

10. Hanchate NK, Giacobini P, Lhuillier $P$, et al. SEMA3A, a Gene involved in axonal pathfinding, is mutated in patients with Kallmann syndrome. PLoS Genet 2012 ; 8 : el002896.

11. Marcos S, Monnier C, Rovira X, et al. Defective signaling through plexin-Al compromises the development of the peripheral olfactory system and neuroendocrine reproductive axis in mice. Hum Mol Genet 2017 ; 26 : 2006-17.

12. Kotan LD, Ternier G, Cakir AD, et al. Loss-of-function mutations in sema3f and plxna3 encoding semaphorin-3F and its receptor Plexin-A3 respectively cause idiopathic hypogonadotropic hypogonadism. Genet Med 2021. doi: 10.1038/s41436-020-01087-5.

13. Van der Klaauw AA, Croizier S, Mendes de Oliveira $\varepsilon$, et al. Human semaphorin 3 variants link melanocortin circuit development and energy balance. Cell $2019 ; 176: 729-42 \mathrm{e} 18$.

14. Wehner AB, Abdesselem H, Dickendesher TL, et al. Semaphorin $3 A$ is a retrograde cell death signal in developing sympathetic neurons. Development $2016 ; 143: 1560-70$.

15. Orr BO, Fetter RD, Davis GW. Retrograde semaphorin-plexin signalling drives homeostatic synaptic plasticity. Nature 2017 ; 550 : 109-13.

16. Howard SR, Guasti L, Ruiz-Babot G, et al. IGSF10 mutations dysregulate gonadotropin-releasing hormone neuronal migration resulting in delayed puberty. EMBO Mol Med $2016 ; 8$ : 626-42.

17. Abreu AP, Dauber A, Macedo DB, et al. Central precocious puberty caused by mutations in the imprinted gene MKRN3. N EnglJ Med 2013 ; 368 : 2467-75.

18. Abreu AP, Toro CA, Song YB, et al. MKRN3 inhibits the reproductive axis through actions in kisspeptin-expressing neurons. J Clin Invest $2020 ; 130:$ 4486-500.

19. Heras V, Sangiao-Alvarellos S, Manfredi-Lozano M, et al. Hypothalamic miR-30 regulates puberty onset via repression of the puberty-suppressing factor, Mkrn3. PLoS Biol 2019; 17 : e3000532.

20. Dauber A, Cunha-Silva M, Macedo DB, et al. Paternally inherited DLKI deletion associated with familial central precocious puberty. J Clin Endocrinol Metab 2017 ; 102 : 1557-67.
21. Silveira LG, Noel SD, Silveira-Neto AP, et al. Mutations of the KISSI gene in disorders of puberty. J Clin Endocrinol Metab 2010 ; 95 : 2276-80.

22. Teles MG, Bianco SD, Brito VN, et al. A GPR54-activating mutation in a patient with central precocious puberty. N Engl J Med 2008 ; 358 : 709-15.

23. Ahmed ML, Ong KK, Morrell DJ, et al. Longitudinal study of leptin concentrations during puberty: sex differences and relationship to changes in body composition. J Clin Endocrinol Metab 1999; $84: 899-905$.

24. Prevot V. Puberty in mice and rats. In : Plant TM, Zeleznik J, eds. Knobil and Neill's physiology of reproduction. New York : Elsevier, 2015 : 1395-439.

25. Tena-Sempere M. Physiological mechanisms for the metabolic control of reproduction. In : Plant TM, Zeleznik J, eds. Knobil and Neill's physiology of reproduction. New York : Elsevier, 2015 : 1605-36.

26. He $Q$, Karlberg J. Bmi in childhood and its association with height gain, timing of puberty, and final height. Pediatr Res $2001 ; 49: 244-51$.

27. McKinney TD, Desjardins C. Postnatal development of the testis, fighting behavior, and fertility in house mice. Biol Reprod 1973 ; $9: 279-94$.

28. Hess RA, Renato de Franca L. Spermatogenesis and cycle of the seminiferpus epithelium. In : Cheng Cy, ed. Molecular mechanisms in spermatogenesis. New York: Springer-Verlag, 2008 : 1-15.

29. McGee SR, Narayan P. Precocious puberty and Leydig cell hyperplasia in male mice with a gain of function mutation in the LH receptor gene. Endocrinology 2013; 154 : 3900-13.

30. Giger RJ, Wolfer DP, De Wit GM, Verhaagen J. Anatomy of rat semaphorin III/ collapsin-1 mRNA expression and relationship to developing nerve tracts during neuroembryogenesis. J Comp Neurol 1996 ; 375 : 378-92.

31. Dluzen DE, Ramirez VD, Carter CS, Getz LL. Male vole urine changes luteinizing hormone-releasing hormone and norepinephrine in female olfactory bulb. Science $1981 ; 212: 573-5$.

32. Boehm U, Zou Z, Buck LB. Feedback loops link odor and pheromone signaling with reproduction. Cell $2005 ; 123: 683-95$.

33. Hellier V, Brock 0 , Candlish $M$, et al. Female sexual behavior in mice is controlled by kisspeptin neurons. Nat Commun $2018 ; 9: 400$.

34. Messina A, Langlet $F$, Chachlaki $K$, et al. A microRNA switch regulates the rise in hypothalamic GnRH production before puberty. Nat Neurosci 2016 ; 19 : 835-44.

35. Oboti L, Trova S, Schellino R, et al. Activity dependent modulation of granule cell survival in the accessory olfactory bulb at puberty. Front Neuroanat $2017 ; 11: 44$

36. Golub MS, Collman GW, Foster PM, et al. Public health implications of altered puberty timing. Pediatrics 2008 ; 121 (suppl 3) : S218-30.

37. Comninos AN, Dhillo WS. Emerging roles of kisspeptin in sexual and emotional brain processing. Neuroendocrinology 2018 ; 106 : 195-202.

38. Ferrero DM, Moeller LM, Osakada T, et al. A juvenile mouse pheromone inhibits sexual behaviour through the vomeronasal system. Nature 2013 ; $502: 368-71$.

39. Gelstein S, Yeshurun Y, Rozenkrantz L, et al. Human tears contain a chemosignal. Science $2011 ; 331: 226-30$.

40. He Z, Tessier-Lavigne M. Neuropilin is a receptor for the axonal chemorepellent Semaphorin III. Cell $1997 ; 90: 739-51$.

41. Kolodkin AL, Levengood DV, Rowe $\varepsilon G$, et al. Neuropilin is a semaphorin III receptor. Cell $1997 ; 90: 753-62$

42. Tamagnone L, Artigiani $\mathrm{S}$, Chen $\mathrm{H}$, et al. Plexins are a large family of receptors for transmembrane, secreted, and GPI-anchored semaphorins in vertebrates. Cell $1999 ; 99: 71-80$.

TIRÉS À PART

V. Prévot

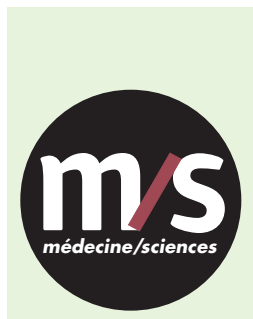

Tarifs d'abonnement $m / s-2021$

> Grâce à $m / s$, vivez en direct les progrès des sciences biologiques et médicales

Abonnez-vous

à médecine/sciences

Abonnez-vous sur www.medecinesciences.org

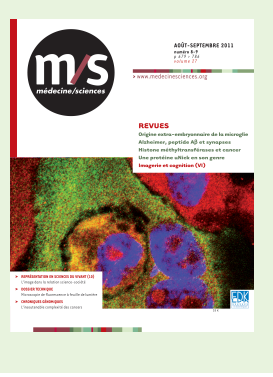

